\title{
ESTUDO TÉRMICO, CARACTERIZAÇÃO ESPECTROSCÓPICA E DIFRAÇÃO DE RAIO X DO COCRISTAL DE ÁCIDO SALICÍLICO COM ÁCIDO NICOTÍNICO OBTIDO POR SÍNTESE MECANOQUÍMICA
}

\author{
Laura Teófilo Ferreira' ${ }^{1}$, Bruno Barreto da Cunha Holanda ${ }^{1}$, Rafael Turra Alarcon ${ }^{1}$, Gilbert Bannach ${ }^{1 \star}$ \\ 1 Faculdade de Ciências, UNESP, Bauru, SP, Brazil. \\ *gilbert@fc.unesp.br
}

\section{Acepted 19/08/2016 \\ Published in 01/03/2017}

\section{Keywords}

Cocristal

Ácido salicílico

Ácido nicotínico

DOI 10.18362/bjta.v6.i1.7

\section{RESUMO}

O objetivo deste trabalho foi formar um novo co-cristal de ácido salicílico (AS) com ácido nicotínico (NCO) por moagem mecanoquímica e estudar seu comportamento térmico, espectroscópicos e difratograma de raio X. Para esta finalidade, 0 método de difração raio $X$ (DRX) pelo método do pó foi utilizado para verificar a diferença de cristalinidade do AS, NCO e o cocristal. Os dados espectroscópicos (FTIR) foram usados para determinar os locais de interação nestas moléculas; bem como Análise Termogravimétrica - Análise Térmica Diferencial (TG-DTA) simultaneas e Calorimetria de Exploratória Diferencial (DSC) foram realizadas para avaliar a estabilidade térmica, etapa de degradação, e eventos térmicos como ponto de fusão e de cristalização. Todas estas técnicas foram utilizadas para verificar a formação do cocristal.

Introdução

A literatura mostra que, atualmente, há um notável e crescente interesse no estudo de compostos denominados "cocristais" tendo em vista suas potencialidades em melhorar algumas propriedades de drogas que já estão inseridas no mercado [1]. A formação de cocristais [2,3] tem sido um importante método de construção de formas cristalinas de moléculas como alternativa a polimorfos ou sólidos multicomponentes, tais como sais, solvatos ou hidratos [4].

Um cocristal farmacêutico pode ser definido como um sólido cristalino multicomponente contendo duas ou mais partes, sendo estas um ingrediente farmacologicamente ativo (API, do inglês "Active Pharmacological Ingredient) neutro e um coformador neutro, unidos por interações não-covalentes como interações de hidrogênio, dipolo-dipolo e , [5-7] sendo uma boa alternativa para melhorar propriedades físico-químicas de um principio ativo, como, por exemplo, sua solubilidade [8], estabilidade $[9,10]$ e, portanto sua biodisponibilidade [11].

Os cocristais podem ser obtidos por diferentes métodos, tais como, mecanoquímico, solvotérmico, cristalização por evaporação lenta de solvente, entre outros [5]. 0 método mecanoquímico, vem se sendo cada vez mais estudado, pois é possível promover reações entre sólidos de forma rápida e quantitativa, sendo assim uma boa opção por utilizar pequena quantidade de reagentes, ser rápido e reprodutível [12].

ELBAGERMA (2010) relata a obtenção do cocristal de ácido nicotínico (NCO) com ácido salicílico (AS) na proporção de 1:1 (mol:mol), obtido por cristalização por evaporação lenta utilizando etanol como solvente [13]. Na literatura são relatados outros trabalhos utilizando diferentes métodos de formação de cocristais com ácido nicotínico ou ácido salicílico [14-17], no entanto, nenhum com a formação de cocristal entre NCO e AS, utilizando método mecanoquímico e em proporção de 1:2

$\mathrm{O}$ ácido nicotínico (niacina) possui dois centros aceptores (-N, $\mathrm{C}=0$ ) e um doador $(-\mathrm{OH})$ para a formação de interações de hidrogênio enquanto 0 ácido salicílico possui dois centros doadores $(-\mathrm{OH})$ e um centro aceptor $(\mathrm{C}=\mathrm{O})$ possibilitando interações de hidrogênio (Figura 1). (a)<smiles>O=C(O)c1ccccc1O</smiles>

(b)<smiles>O=C(O)c1cccnc1</smiles>

Figura 1. Fórmula estrutural dos reagentes: (a) Ácido Salicílico e (b) Ácido Nicotínico.

Materiais e métodos

\section{Síntese Mecanoquímica}

0 ácido salicílico e o ácido nicotínico foram comprados na Sigma-Aldrich com pureza $\geq 98 \%$. Realizou-se a síntese mecanoquímica em moinho de bolas MM40, com 2 (duas) esferas de aço inox, em uma frequência de moagem de $30 \mathrm{~Hz}$ 
durante 1 hora, sem solvente. Utilizou-se massa de 0,2762 g de AS e 0,1231 g de NCO na proporção de 2:1 (AS:NCO) mol:mol.

\section{Análise térmica (TG-DTA e DSC)}

As curvas TG-DTA foram obtidas pelo equipamento Netzsch modelo STA 449 F3, utilizou-se massa de amostras de aproximadamente $5 \mathrm{mg}$ e razão de aquecimento de $20,0^{\circ} \mathrm{C} \cdot \mathrm{min}^{-1}$ em atmosfera de ar seco com vazão de $50 \mathrm{~mL} \cdot \mathrm{min}^{-1}$ e intervalo de temperatura de $30,0^{\circ} \mathrm{C}$ a $800,0^{\circ} \mathrm{C}$.

As curvas DSC e imagens foram obtidos pelo equipamento Mettler-Toledo modelo DSC 1 Stare System com câmera digital SC30 acoplada, que incorpora um sensor CMOS de 3,3 megapixel, subconjunto ótico mecânico Navitar 1-6232D com zoom de 6,5X. Utilizou-se massa de amostras de aproximadamente $2 \mathrm{mg}$, razão de aquecimento de $10,0^{\circ} \mathrm{C} \cdot \mathrm{min}^{-1}$ em atmosfera de ar seco com vazão de $50,0 \mathrm{~mL} \mathrm{~min}^{-1}$, com intervalo de aquecimento de acordo com a estabilidade térmica de cada composto. Em cadinho de alumínio, 40 I, com tampa prensada e perfurada e cadinho de -alumina aberto, $30 \mathrm{I}$, para filmagem do processo de aquecimento. O DSC cíclico do cocristal também foi realizada, nos intervalos de temperatura de $25,0^{\circ} \mathrm{C}$ até $160,0^{\circ} \mathrm{C}$ e $160,0^{\circ} \mathrm{C}$ até $25,0^{\circ} \mathrm{C}$ utilizando a massa de amostra descrita acima.

\section{Espectroscopia de Infravermelho com} Transformada de Fourier (FTIR)

Os espectros de absorção na região do infravermelho médio foram obtidos pelo espectrômetro Vertex 70 , da Bruker. Os espectros foram obtidos através do método de refletância atenuada (ATR), com faixa de varredura entre $4000 \mathrm{~cm}^{-1}$ a 400 $\mathrm{cm}^{-1}$ (resolução de $4 \mathrm{~cm}^{-1}$ ) com cristal de diamante como suporte.

\section{DRX pelo método do pó}

Os difratogramas de raios $X$ foram obtidos pelo Difratômetro Siemens DMAX 2000 utilizando-se tubo de cobre, submetido a $40 \mathrm{kV}$, corrente de $20 \mathrm{~mA}, \mathrm{Cu} \mathrm{Ka}, \lambda=1,5406 \AA$. As amostras foram colocadas em suporte de vidro, e expostas à radiação $\left(5^{\circ} \leq 2 \theta \leq 50^{\circ}\right)$.

\section{Resultados e discussão}

As curvas TG-DTA do NCO, AS e do cocristal podem ser observados na Figura 2. Observa-se na curva DTA um pico endotérmico, em $230,8^{\circ} \mathrm{C}$, referente à fusão do $\mathrm{NCO}$. As curvas mostram que o NCO é estável até $189,0{ }^{\circ} \mathrm{C}$, temperatura referente ao início do processo de fusão, evaporando em seguida $(\Delta m=100 \%)$, etapa associada ao pico endotérmico em $311,1^{\circ} \mathrm{C}$. O AS é estável até $145,1^{\circ} \mathrm{C}$, temperatura referente ao início do processo de fusão, evaporando em seguida $(\Delta \mathrm{m}=$ $100 \%$ ), etapa associada ao pico endotérmico em $341,2{ }^{\circ} \mathrm{C}$. $\mathrm{O}$ cocristal apresenta uma estabilidade menor que os compostos puros $\left(130,0^{\circ} \mathrm{C}\right)$, e se decompõe em seguida, em uma única etapa associada ao pico exotérmico em $202,0^{\circ} \mathrm{C}$ na curva DTA.

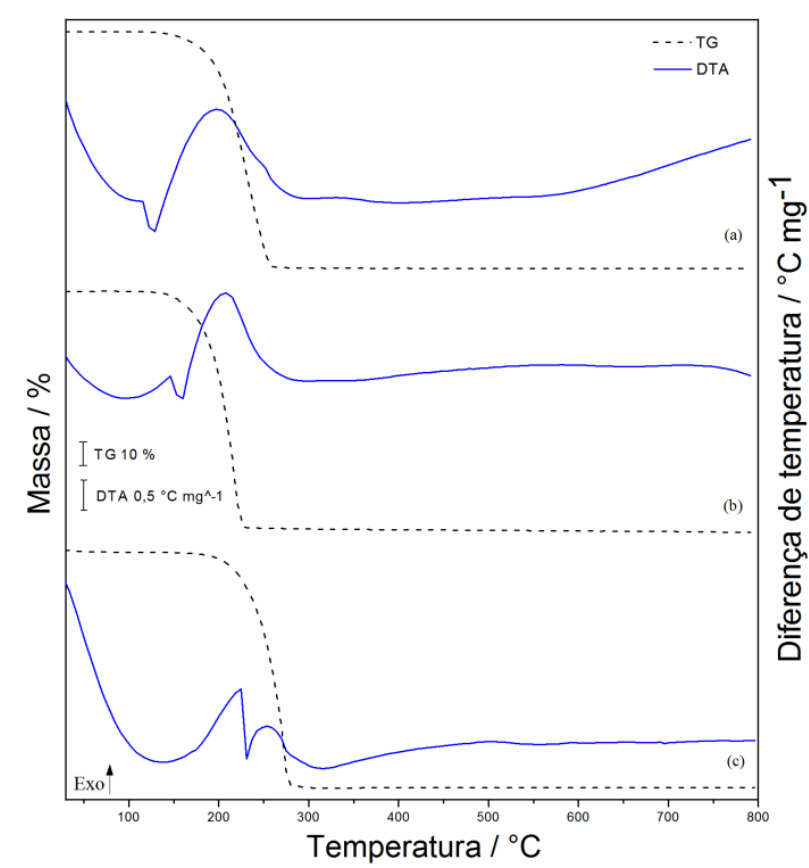

Figura 2. TG-DTA do (a) cocristal, (b) ácido salicílico, (c) ácido nicotínico

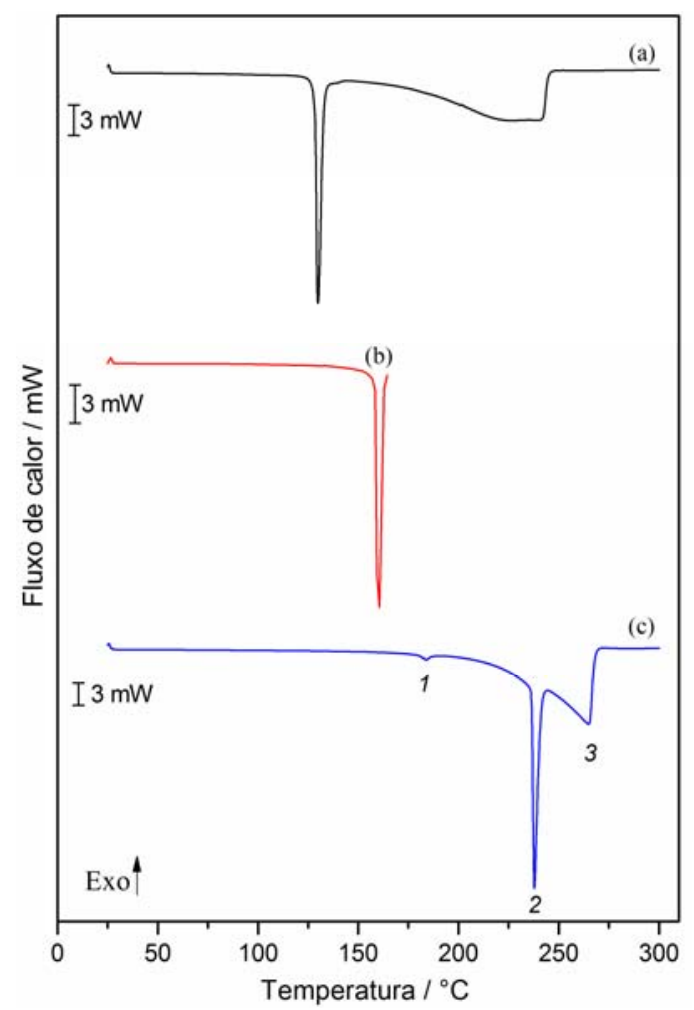

Figura 3. DSC do (a) cocristal, (b) ácido salicílico, (c) ácido nicotínico

Na Figura 3 são apresentadas as curvas DSC, as medidas foram realizadas com base na estabilidade térmica dos compostos. Observa-se nas curvas DSC (Figura 3) que o NCO apresenta um pequeno pico endotérmico (1) em $183,4{ }^{\circ} \mathrm{C}$, 
Braz. J. Therm. Anal. Vol. 6 No. 1

atribuído a uma transição de fase sólido-sólido [18] um pico endotérmico (2), referente à fusão, em $237,8^{\circ} \mathrm{C}$, seguindo por um evento endotérmico (3) referente à evaporação do composto, como pode ser observado nas imagens obtidas pelo DSCFotovisual na Figura 4. O AS apresenta um pico endotérmico de fusão em $160{ }^{\circ} \mathrm{C}$ e o cocristal apresenta um pico endotérmico referente à fusão em $129,7^{\circ} \mathrm{C}$ e uma endoterma entre $170{ }^{\circ} \mathrm{C} \mathrm{e}$ $250^{\circ} \mathrm{C}$, referente à evaporação do cocristal, na Figura 5 as imagens obtidas pelo DSC-Fotovisual de (a) a (c) mostram a fusão e as de (d) a (f) mostram a evaporação do cocristal. No DSC cíclico, Figura 6, observa-se a fusão do cocristal em 129,7 ${ }^{\circ} \mathrm{C}$ e um pico exotérmico, durante o resfriamento, em $72,3^{\circ} \mathrm{C}$, referente à cristalização, como pode ser observado nas imagens obtidas pelo DSC-Fotovisual na Figura 7.

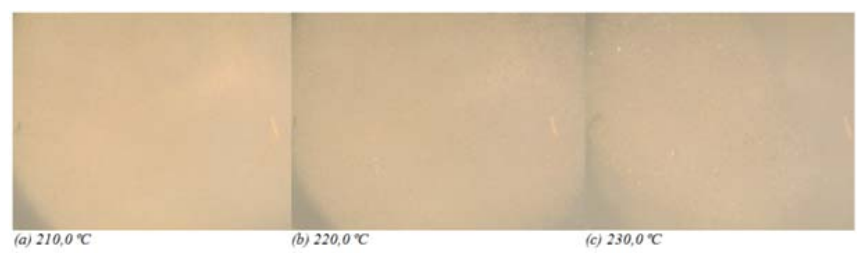

Figura 4. DSC - Fotovisual evaporação do NCO
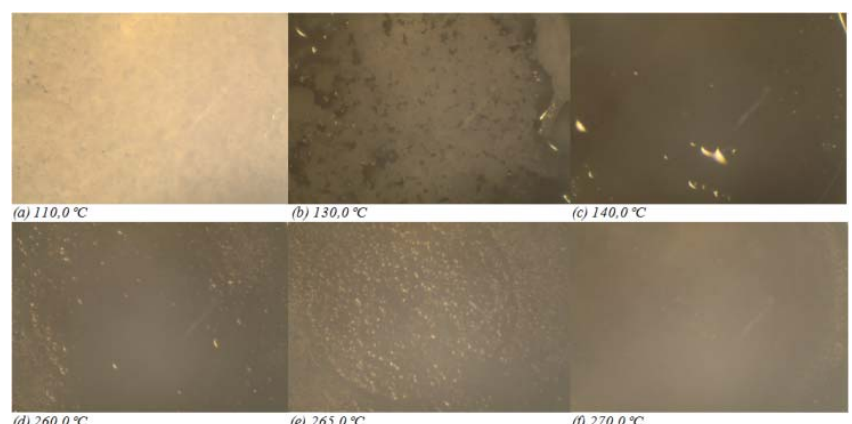

Figura 5. DSC da fusão e evaporação do cocristal

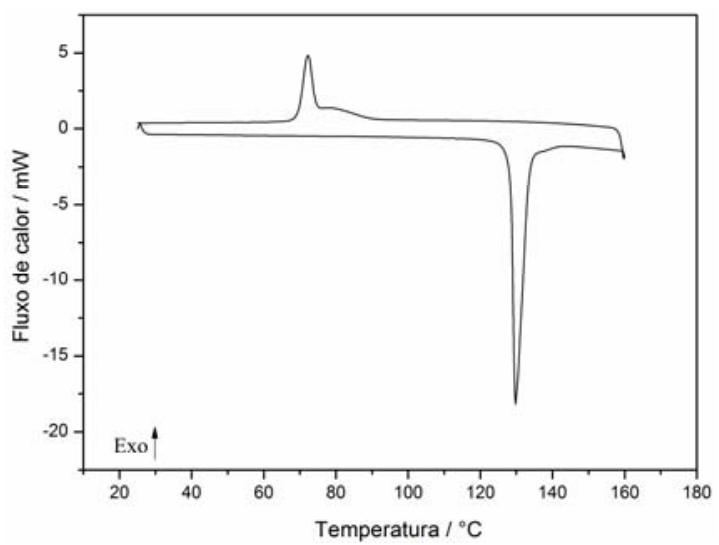

Figura 6. DSC cíclico do cocristal

\section{DOI 10.18362/bjta.v6.i1}

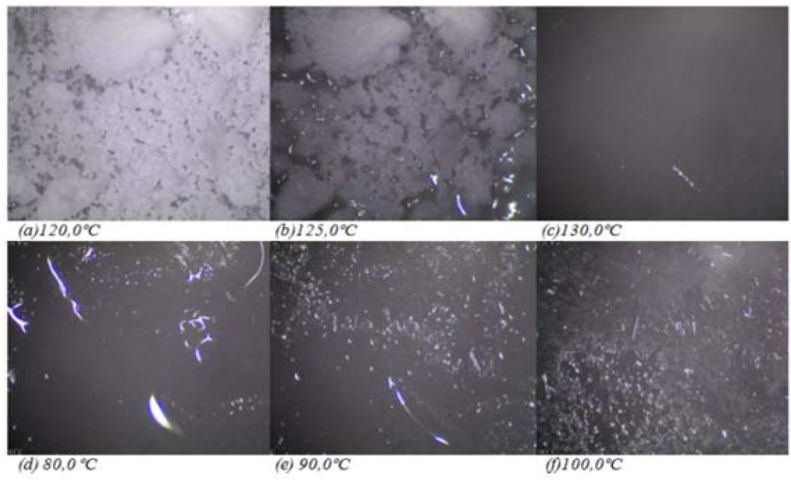

Figura 7. DSC - Fusão e cristalização do cocristal

No difratograma de raios $X$ do cocristal, podem-se observar picos diferentes daqueles que são observados nos difratogramas do NCO e do AS puros e a ausência de alguns picos destes, confirmando assim à formação de um cocristal. As principais modificações podem ser observadas pelo aparecimento dos picos de difração em $7,52^{\circ} ; 12,68^{\circ} ; 13,24^{\circ}$, $14,88^{\circ}, 18,92^{\circ}, 19,56^{\circ}, 29,88^{\circ}$ e $43,80^{\circ}$ no difratograma do cocristal. Os picos destacados no difratograma do cocristal (Figura 8 (a)) são os picos que não são observados nos difratogramas dos compostos puros. Os picos destacados nos compostos puros $\left(15,80^{\circ} ; 46,88^{\circ}\right.$; e $47,24^{\circ}$ difratograma de raios $X$ do AS) $\left(21,08^{\circ} ; 25,88^{\circ} ; 26,80^{\circ} ; 34,04^{\circ} ; 41,00^{\circ}\right.$ e $41,36^{\circ}$ no difratograma de raios $X$ do NCO) não aparecem no difratograma do cocristal.

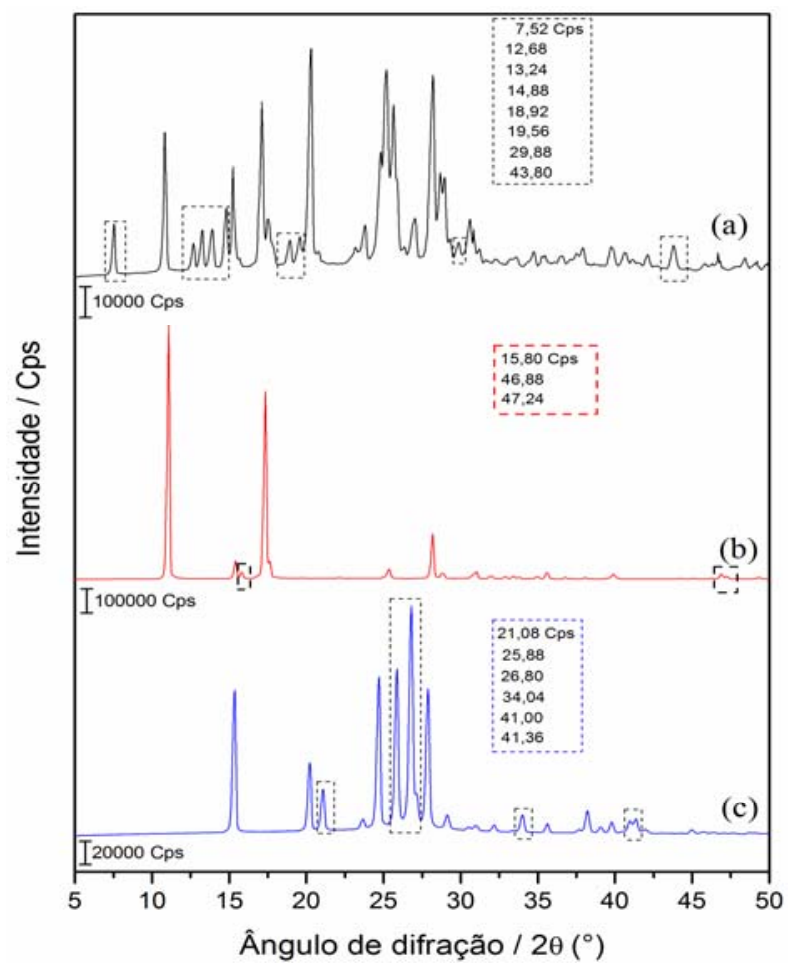

Figura 8. Difratogramas de raio $X$ do (a) cocristal, (b) ácido salicílico, (c) ácido nicotínico 
A técnica de espectrometria de absorção na região média do infravermelho foi utilizada com o objetivo de verificar possíveis interações entre os grupos funcionais do NCO e AS. Esta comparação pode mostrar indicações se há obtenção de um cocristal ou somente o espectro soma dos constituintes.

Analisando a Figura 9, pode-se verificar que no espectro cocristal a ausência das bandas $C=0\left(1702 \mathrm{~cm}^{-1}\right) \mathrm{e}$ Naromático $\left(1415 \mathrm{~cm}^{-1}\right)$ e do NCO, indicando que o ácido nicotínico participa da ligação de hidrogênio, e da banda $\mathrm{O}-\mathrm{H}\left(3230 \mathrm{~cm}^{-1}\right)$ do AS confirmando a proporção estequiometria do cocristal (2 AS : $1 \mathrm{NCO}$ ). Utilizando os dados obtidos pelos espectros podem-se propor os síntons formados neste cocristal, Figura 10, heterosínton ácido-piridina e homosínton ácido-ácido.

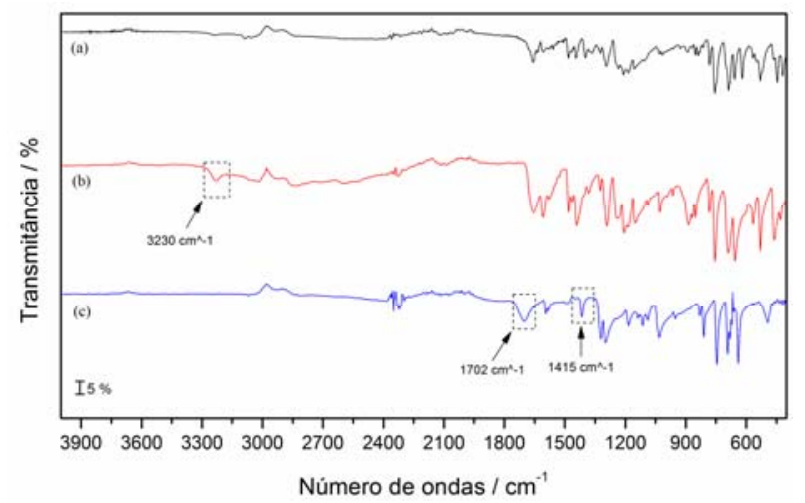

Figura 9. FTIR do (a) cocristal, (b) ácido salicílico, (c) ácido nicotínico

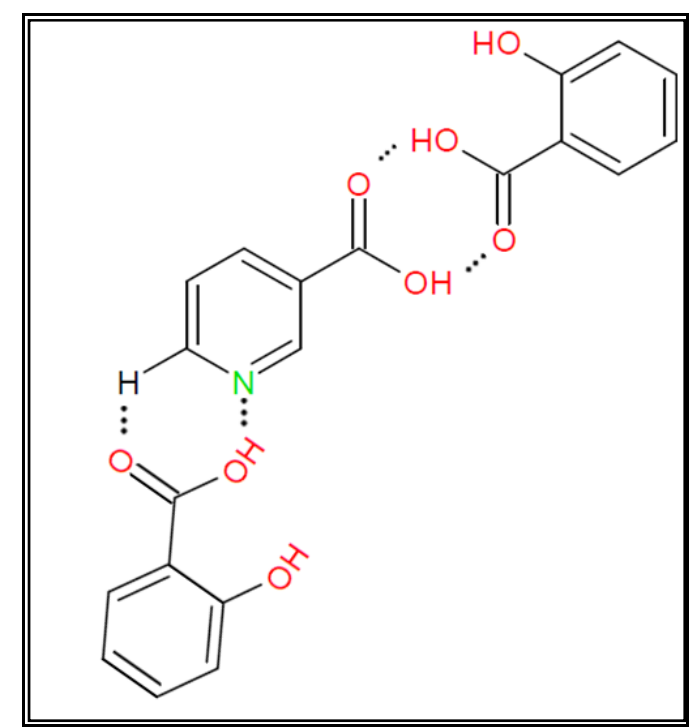

Figura 10. Síntons supramoleculares propostos para o cocristal

\section{Conclusão}

A existência do cocristal de ácido salicílico com ácido nicotínico na proporção de 2:1 (mol:mol), obtido por síntese mecanoquímica sem solvente, pôde ser confirmada utilizando-se as técnicas de difração de raio $X$ pelo método do pó, infravermelho médio e análise térmica (TG-DTA e DSC). Os estudos térmicos do cocristal e do ácido nicotínico mostram eventos térmicos importantes tais como estabilidade térmica, fusão, cristalização, transição de fase sólido-sólido e decomposição, muitas vezes confirmados pelas imagens obtidas pelo DSC-Fotovisual. Alterações de bandas no espectro de infravermelho médio são um importante diagnóstico de formação de cocristal, e os resultados obtidos sugerem interação de hidrogênio entre API e co-formador. 0 método de difração de raios $X$ pelo pó mostra que o processo de moagem resultou em uma nova estrutura cristalina para o cocristal diferente dos compostos puros.

\section{Agradecimentos}

Faculdade de Ciências -UNESP Campus Bauru

PIBIC - CNPq (Processo 116209/2016-0)

FAPESP (Processos 2013/09022-7)

CAPES (Processo 024/2012 Pró-equipamentos)

\section{Referencias}

[1] Sekhon, B. S. (2009). Pharmaceutical co-crystals-a review.

[2] Steed, J. W. (2013). The role of co-crystals in pharmaceutical design. Trends in pharmacological sciences, 34(3), 185193.

[3] Trask, A. V., Motherwell, W. S., \& Jones, W. (2006). Physical stability enhancement of theophylline via cocrystallization. International journal of pharmaceutics, 320(1), 114-123.

[4] Friščić, T., Childs, S. L., Rizvi, S. A., \& Jones, W. (2009). The role of solvent in mechanochemical and sonochemical cocrystal formation: a solubility-based approach for predicting cocrystallisation outcome. CrystEngComm, 11(3), 418-426.

[5] Kuminek, G., Cao, F., da Rocha, A. B. D. O., Cardoso, S. G., \& Rodríguez-Hornedo, N. (2016). Cocrystals to facilitate delivery of poorly soluble compounds beyond-rule-of-5. Advanced drug delivery reviews, 101, 143-166.

[6] Prado, L. D., \& Rocha, H. V. A. (2015). Estado Sólido na Indústria Farmacêutica: Uma Breve Revisão. Revista Virtual de Química, 7(6), 2080-2112.

[7] Lara-Ochoa, F., \& Espinosa-Perez, G. (2007). Crystals and patents. Crystal growth \& design, 7(7), 1213-1215.

[8] Good, D. J., \& Rodríguez-Hornedo, N. (2009). Solubility advantage of pharmaceutical cocrystals. Crystal Growth and Design, 9(5), 2252-2264.

[9] Trask, A. V., Motherwell, W. S., \& Jones, W. (2006). Physical stability enhancement of theophylline via cocrystallization. International journal of pharmaceutics, 320(1), 114-123.

[10] Manin, A. N., Voronin, A. P., Shishkina, A. V., Vener, M. V., Churakov, A. V., \& Perlovich, G. L. (2015). Influence of Secondary Interactions on the Structure, Sublimation Thermodynamics, and Solubility of Salicylate: 4- 
Hydroxybenzamide Cocrystals. Combined Experimental and Theoretical Study. The Journal of Physical Chemistry B, 119(33), 10466-10477.

[11] McNamara, D. P., Childs, S. L., Giordano, J., larriccio, A., Cassidy, J., Shet, M. S., ... \& Park, A. (2006). Use of a glutaric acid cocrystal to improve oral bioavailability of a low solubility API. Pharmaceutical research, 23(8), 18881897

[12] Hasa, D., Schneider Rauber, G., Voinovich, D., \& Jones, W. (2015). Cocrystal Formation through Mechanochemistry: from Neat and Liquid-Assisted Grinding to PolymerAssisted Grinding. Angewandte Chemie International Edition, 54(25), 7371-7375.

[13] Elbagerma, M. A., Edwards, H. G. M., Munshi, T., Hargreaves, M. D., Matousek, P., \& Scowen, I. J. (2010). Characterization of new cocrystals by Raman spectroscopy, powder X-ray diffraction, differential scanning calorimetry, and transmission Raman spectroscopy. Crystal Growth \& Design, 10(5), 2360-2371.

[14] Arenas-Garcia, J. I., Herrera-Ruiz, D., Mondragón-Vásquez, K., Morales-Rojas, H., \& Höpfl, H. (2011). Modification of the supramolecular hydrogen-bonding patterns of acetazolamide in the presence of different cocrystal formers: $3: 1,2: 1,1: 1$, and $1: 2$ cocrystals from screening with the structural isomers of hydroxybenzoic acids, aminobenzoic acids, hydroxybenzamides, aminobenzamides, nicotinic acids, nicotinamides, and 2, 3dihydroxybenzoic acids. Crystal Growth \& Design, 12(2), 811-824.

[15] Perman, J. A., Dubois, K., Nouar, F., Zoccali, S., Wojtas, Ł., Eddaoudi, M., ... \& Zaworotko, M. J. (2009). Cocrystal Controlled Solid-State Synthesis of a Thermally Stable Nicotinate Analogue That Sustains an Isostructural Series of Porous Metal- Organic Materials. Crystal Growth \& Design, 9(12), 5021-5023.

[16] Zhou, Z., Chan, H. M., Sung, H. H. Y., Tong, H. H., \& Zheng, Y. (2016). Identification of New Cocrystal Systems with Stoichiometric Diversity of Salicylic Acid Using Thermal Methods. Pharmaceutical research, 33(4), 1030-1039.

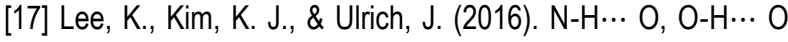
hydrogen bonded supramolecular formation in the cocrystal of salicylic acid with $\mathrm{N}$-containing bases. Crystal Research and Technology, 51(3), 197-206.

[18] Wang, S. X., Tan, Z. C., Di, Y. Y., Xu, F., Wang, M. H., Sun, L. X., \& Zhang, T. (2004). Calorimetric study and thermal analysis of crystalline nicotinic acid. Journal of thermal analysis and calorimetry, 76(1), 335-342. 